\title{
Hop Hubtic Number and Hop Hub Polynomial of Graphs
}

\author{
Abdu-Alkafi Saead Sand \\ Department of Mathematics \\ Faculty of Education and Science \\ Al-Baydha University, Yemen.
}

\author{
Sultan Senan Mahde \\ Department of Mathematics \\ Faculty of Education and Science \\ Al-Baydha University, Yemen.
}

\begin{abstract}
The maximum order of partition of the vertex set $V(G)$ into vertex hop hub sets is called hop hubtic number of $G$ and denoted by $h_{\xi}(G)$. In this paper the hop hubtic number of some standard graphs was determined. Also bounds for $h_{\xi}(G)$ were obtained. The hop hub polynomial of a connected graph $G$ was introduced. The hop hub polynomial of a connected graph $G$ of order $n$ is the polynomial

$$
H_{h}(G, x)=\sum_{i=h_{h}(G)}^{|V(G)|} h_{h}(G, i) x^{i},
$$

where $h_{h}(G, i)$ denotes the number of hop hub sets of $G$ of cardinality $i$ and $h_{h}(G)$ is the hop hub number of $G$. Finally, the hop hub polynomial of some special classes of graphs was studied.
\end{abstract}

\section{General Terms}

AMS, Subject Classification 05C40, 05C99.

\section{Keywords}

Hubtic number, Hop Hubtic number, Hop Hub number, Hub polynomial.

\section{INTRODUCTION}

By a graph $G=(V, E)$ we mean a finite and undirected graph without loops and multiple edges. A graph $G$ with $n$ vertices and $m$ edges is called a $(n, m)$ graph, the number $n$ is referred to as the order of a graph $G$ and $m$ is referred to as the size of a graph $G$. In general, the degree of a vertex $v$ in a graph $G$ denoted by $\operatorname{deg}(v)$ is the number of edges of $G$ incident with $v$. Also $\delta(G)$ denotes the minimum degree among the vertices of $G[5]$. In a tree, a leaf is a vertex of degree one. See [5] for terminology and notations not defined here.

A set $D$ of vertices in a graph $G$ is called dominating set of $G$ if every vertex in $V-D$ is adjacent to some vertex in $D$, the minimum cardinality of a dominating set in $G$ is called the domination number $\gamma(G)$ of a graph $G$ [6]. In 1977, E. J. Cockayne and S. T. Hedetniemi have introduced the concept of domatic number of graph $G$ and defined by, a $D$-partition of $G$ is a partition of $V(G)$ into dominating sets, the domatic number of $G$ denoted by $d(G)$ is the maximum order of a $D$-partition of $G[3]$.
Introduced by Walsh [14], a hub set in a graph $G$ is a set $H$ of vertices in $G$ such that any two vertices outside $H$ are connected by a path whose all internal vertices lie in $H$. The hub number of $G$, denoted by $h(G)$, is the minimum size of a hub set in $G$. Shadi, Veena, and Sultan [7] have introduce the maximum order of partition of the vertex set $V(G)$ into hub sets is called hubtic number of $G$, and denoted by $\xi(G)$. A $H$-partition of a graph $G$ is a partition of $V(G)$ into hub sets. There are many polynomials associated with graphs. For example, domination polynomial, chromatic polynomial, clique polynomial, characteristic polynomial and Tutte polynomial, see [1, 2, 4, 10 12]. In 2020, R. P. Veettil and T. V. Ramakrishnan [13] introduce hub polynomial of a connected graph G. The hub polynomial of a connected graph $G$ of order $n$ is the polynomial

$$
H_{G}(x)=\sum_{i=h(G)}^{|V(G)|} h(G, i) x^{i}
$$

where $h(G, i)$ denotes the number of hub sets of $\mathrm{G}$ of cardinality $i$ and $h$ is the hub number of $G$. In 2020, Sultan and Veena [8] defined hub-integrity polynomial of graphs and obtained some of its properties. Also in 2021, S. S. Mahde and A. S. Sand have introduced the concept of hop hub integrity [9].

In 2021, A. S. Sand and S. S. Mahde [11], have introduced the concept of hop hub set of a graph which is defined as follows. A hub set $S$ is a hop hub set of $G$ if for every $v \in V-S$, there exists $u \in S$ such that $d(u, v)=2$. The minimum cardinality of a hop hub set of $G$ is called the hop hub number and is denoted by $h_{h}(G)$. Using the concept of hop hub set of a graph $G$ and the definition of the hubtic number of a graph $G$, motivated by this, we introduce the concept of hop hubtic number of a graph $G$ as a new parameter of a graph.

The following results will be useful in the proof of our results. 1.

Proposition 1. ([3]) For any graph $G$, $\operatorname{daim}(G) \leq \delta(G)+$

LEMMA 1. ([11]) Let $T$ be a tree with $n$ vertices and l leaves and $p$ internal vertices, then $h_{h}(T)=h(T)=n-l$ such that $p \geq 3$.

PROPOSITION 2. ([11]) The hop hub numbers of some specific classes of graphs are as below: 
(1) For any path $P_{n}$,

$$
h_{h}\left(P_{n}\right)= \begin{cases}2, & \text { if } n=2, \\ 3, & \text { if } n=3, \\ n-2, & \text { if } n \geq 4\end{cases}
$$

(2) For any complete graph $K_{n}, h_{h}\left(K_{n}\right)=n$.

(3) For the wheel graph $W_{1, n-1}$,

$$
h_{h}\left(W_{1, n-1}\right)=\left\{\begin{array}{l}
4, \text { if } n=4, \\
3, \text { if } n \geq 5,
\end{array}\right.
$$

(4) For the complete bipartite graph $K_{n, m}, h_{h}\left(K_{n, m}\right)=2$.

(5) For the double star $S_{n, m}, h_{h}\left(S_{n, m}\right)=2$.

(6) For any cycle $C_{n}$

$$
h_{h}\left(C_{n}\right)= \begin{cases}2, & \text { if } n=4, \\ 3, & \text { if } n=3, \\ n-3, & \text { if } n \geq 5\end{cases}
$$

\section{HOP HUBTIC NUMBER OF GRAPHS}

DEFINITION 2. The maximum order of partition of the vertex set $V(G)$ into hop hub sets is called hop hubtic number of $G$, and denoted by $h_{\xi}(G)$. A $H_{h}$-partition of a graph $G$ is a partition of $V(G)$ into hop hub sets.

EXAMPLE 1. The Figure 1] shows a hop hubtic partition of a graph. The hop hub sets $S_{1}=\left\{v_{2}, v_{3}\right\}$, and $S_{2}=\left\{v_{1}, v_{4}, v_{5}, v_{6}\right\}$, are hop hub sets of $G$, so $h_{\xi}(G)=2$.

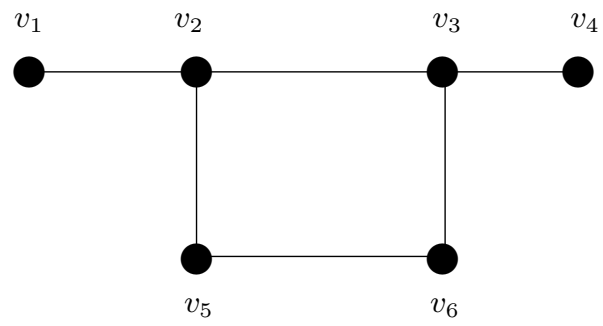

Fig. 1: Graph $(G)$

THEOREM 3. For any connected graph $G, 1 \leq h_{\xi}(G) \leq$ $\left\lfloor\frac{n}{h_{h}(G)}\right\rfloor$.

Proof. Let $H=\left\{H_{1}, H_{2}, H_{3}, \ldots, H_{h_{\xi}(G)}\right\}$ be the hop hubtic partition of graph $G$. Clearly, $|H i| \geq h_{h}(G)$ for all $i=$ $1,2, \ldots, h_{\xi}(G)$, so $h_{\xi}(G)|H i| \geq h_{\xi}(G) h_{h}(G)$ for all $i=$ $1,2, \ldots, h_{\xi}(G)$, then

$$
n=\Sigma|H i| \geq h_{\xi}(G) h_{h}(G) .
$$

Hence the assertion follows.

By Theorem 3 we get the next result.

Proposition 3. (1) For any complete graph $K_{n}, h_{\xi}\left(K_{n}\right)=$ 1.

(2) For any path $P_{n}$ with $n \geq 5, h_{\xi}\left(P_{n}\right)=1$.

(3) For the wheel graph $W_{1, n-1}, h_{\xi}\left(W_{1, n-1}\right)=1$.

(4) For the complete bipartite graph $K_{n, m}, h_{\xi}\left(K_{n, m}\right)=$ $\min \{n, m\}$.

(5) For the double star $S_{n, m}, h_{\xi}\left(S_{n, m}\right)=2$.
(6) For any cycle $C_{n}$,

$$
h_{\xi}\left(C_{n}\right)=\left\{\begin{array}{l}
2, \text { if } n=4,5,6 . \\
1, \text { if } n \geq 7 .
\end{array}\right.
$$

Proof. 1- By Theorem 3 and Proposition 2 we have

$$
\begin{aligned}
h_{\xi}\left(K_{n}\right) & \leq\left\lfloor\frac{n}{h_{h}\left(K_{n}\right)}\right\rfloor \\
& =\left\lfloor\frac{n}{n}\right\rfloor \\
& =\lfloor 1\rfloor \\
& =1 \\
h_{\xi}\left(K_{n}\right) & \leq 1 .
\end{aligned}
$$

And for any graph $G, h_{\xi}\left(K_{n}\right) \geq 1$, then $h_{\xi}\left(K_{n}\right)=1$. 2- By Theorem 3 , and Proposition 2

$$
\begin{array}{cc}
h_{\xi}\left(P_{n}\right) \quad \leq\left\lfloor\frac{n}{h_{h}\left(P_{n}\right)}\right\rfloor \\
=\left\lfloor\frac{n}{n-2}\right\rfloor \\
=\left\lfloor\frac{n-2+2}{n-2}\right\rfloor \\
=\left\lfloor\frac{n-2}{n-2}+\frac{2}{n-2}\right\rfloor \\
=\left\lfloor 1+\frac{2}{n-2}\right\rfloor \\
=1+\left\lfloor\frac{2}{n-2}\right\rfloor, n \geq 5 \\
=1+0,\left\lfloor\frac{2}{n-2}\right\rfloor=0, n \geq 5 . \\
=1 \\
h_{\xi}\left(P_{n}\right) \quad \leq 1 .
\end{array}
$$

And for any graph $G, h_{\xi}\left(P_{n}\right) \geq 1$, then $h_{\xi}\left(P_{n}\right)=1$ if $n \geq 5$.

3- If $n=4, W_{1,3} \cong K_{4}$ and $h_{\xi}\left(K_{4}\right)=1$ form Proposition 3 . part 1.

If $n \geq 5$, let $V\left(W_{1, n-1}\right)=\left\{v, v_{1}, v_{2}, \cdots, v_{n-1}\right\}$ and $h_{h}\left(W_{1, n-1}\right)=3$, since $v$ is center of $W_{1, n-1}$ adjacent any vertex in $W_{1, n-1}$, then $h_{\xi}\left(W_{1, n-1}\right)=1$.

4- Let $V\left(K_{n, m}\right)=\left\{v_{1}, v_{2}, \ldots, v_{n}, u_{1}, u_{2}, \ldots, u_{m}\right\}$. Consider $H_{h}=\left\{v_{1}, u_{1}\right\}$ is a hop hub set of $K_{n, m}$ such that $\left|H_{h}\right|=2$, therefore any hop hub set $H_{h}$ must contain $\left\{v_{i}, u_{j}\right\}$ such that $i \in n, j \in m$, therefore, the number of hop hub set depended on the minimum vertex $i$ or $j$.

5- Let $V\left(S_{n, m}\right)=\left\{v, v_{1}, v_{2}, \ldots, v_{n}, u, u_{1}, u_{2}, \ldots, u_{m}\right\}$. There are two $H_{h}$-partition of $V\left(S_{n, m}\right)$ are $S_{1}=\{v, u\}$ and $S_{2}=\left\{v_{1}, v_{2}, \ldots, v_{n}, u_{1}, u_{2}, \ldots, u_{m}\right\}$, then $h_{\xi}\left(S_{n, m}\right)=2$

6- Consider $V\left(C_{n}\right)=\left\{v_{1}, v_{2}, \ldots, v_{n}\right\}$ be the vertex of cycle, the following cases are discussed:

Case 1: When $n=4$, there only two $H_{h}$ sets of cardinality two, namely, $S_{1}=\left\{v_{1}, v_{2}\right\}$ and $S_{2}=\left\{v_{3}, v_{4}\right\}$. Therefore, $h_{\xi}\left(C_{4}\right)=2$.

Case 2: When $n=5$, there only one $H_{h}$ set of cardinality two, namely, $S_{1}=\left\{v_{1}, v_{2}\right\}$ and one set of cardinality three, namely, $S_{2}=\left\{v_{3}, v_{4}, v_{5}\right\}$. Then, $h_{\xi}\left(C_{5}\right)=2$.

Case 3: When $n=6$, there only two $H_{h}$ sets of cardinality three, namely, $S_{1}=\left\{v_{1}, v_{2}, v_{3}\right\}$ and $S_{2}=\left\{v_{4}, v_{5}, v_{6}\right\}$. So, $h_{\xi}\left(C_{6}\right)=2$.

Case 4: When $n \geq 7$. By Theorem 3, and Propositior 2, we have 


$$
\begin{array}{cc}
h_{\xi}\left(C_{n}\right) \quad \leq\left\lfloor\frac{n}{h_{h}\left(C_{n}\right)}\right\rfloor \\
=\left\lfloor\frac{n}{n-3}\right\rfloor \\
=\left\lfloor\frac{n-3+3}{n-3}\right\rfloor \\
=\left\lfloor\frac{n-3}{n-3}+\frac{3}{n-3}\right\rfloor \\
=\left\lfloor 1+\frac{3}{n-3}\right\rfloor \\
=1+\left\lfloor\frac{3}{n-3}\right\rfloor \\
=1+0 . & \left\lfloor\frac{3}{n-3}\right\rfloor=0, \quad n \geq 7 . \\
& =1 \\
h_{\xi}\left(C_{n}\right) & \leq 1 .
\end{array}
$$

And for any graph $G, h_{\xi}\left(C_{n}\right) \geq 1$, so $h_{\xi}\left(C_{n}\right)=1$.

THEOREM 4. Let $G$ be a tree with at least 3 non-leaf vertices. Then $h_{\xi}(G)=1$.

Proof. Suppose that $G$ is a tree with at least 3 non-leaf vertices. We discuss the following cases:

Case 1. Let $H$ be a set of all non-leaf vertices. Clearly, any path between two leaves does not pass through another leaf. So, $H$ is a hop hub set of $G$, and by Lamma 1 it is a minimum hop hub set. Now, suppose that $D \subseteq V(G)-\bar{H}$ is a hop hub set of $G$. Since $G$ is a tree with at least 3 non-leaf vertices, take any two non-adjacent vertices $u, v \in H$. Since all vertices in $D$ are leaves, then there is no path between $u$ and $v$ with all internal vertices in $D$. This is a contradiction. Hence $h_{\xi}(G)=1$.

Case 2. Suppose that $H$ is a hop hub set of $G$ but not containing all non-leaf vertices. Since $G$ has at least three non-leaf vertices, let $\left\{v_{1}, v_{2}, v_{3}\right\}$ be non-leaf vertices and $v_{1} v_{3} \notin E(G)$, let $l_{1}, l_{3}$ be leaves adjacent to $v_{1}$ and $v_{3}$, respectively. Clearly, $G\left[\left\{l_{1}, v_{1}, v_{2}, v_{3}, l_{3}\right\}\right]$ is a path $P_{5}$. Since $h_{h}\left(P_{5}\right)=3$, then $H$ contains at least three vertices from $P_{5}$. Then any other hop hub set of $G$ must intersects $H$ since $\left|P_{5}\right|=5$, therefore $h_{\xi}(G)=1$.

THEOREM 5. For any graph $G, h_{\xi}(G) \leq \delta(G)+1$.

Proof. Suppose $h_{\xi}(G)>\delta(G)+1$, We have the following cases :

Case 1: If $G \cong K_{n}$

$$
\begin{aligned}
h_{\xi}\left(K_{n}\right) & >\delta\left(K_{n}\right)+1 \\
1 & >\delta\left(K_{n}\right)+1 \\
1-1 & >\delta\left(K_{n}\right) \\
0 & >\delta\left(K_{n}\right) .
\end{aligned}
$$

impossible

Case 2: If $G$ is tree, hop hubtic of tree is 1 or 2 by Theorem 4

If hop hubtic of tree is 1 and since $\delta(T)=1$, then

$$
\begin{array}{cc}
h_{\xi}(T) & >1+1 \\
h_{\xi}(T) & >2 \\
1 & >2,
\end{array}
$$

impossible.

If hop hubtic of tree is 2 , then

$$
\begin{aligned}
& 2>1+1 \\
& 2>2
\end{aligned}
$$

this is impossible.

Case 3: Now If $G$ is not tree, then there exist some graph such that
$h_{\xi}(G)=\delta(G)$, so the relation $h_{\xi}(G)>\delta(G)+1$ is not true Therefore, $h_{\xi}(G) \leq \delta(G)+1$.

Lemma 6. For any graph $G, h_{\xi}(G)+\operatorname{daim}(G) \leq 2 \delta(G)+2$.

Proof. By Proposition 1 daim $(G) \leq \delta(G)+1$ and Theorem 5. $h_{\xi}(G) \leq \delta(G)+1$, then $h_{\xi}(G)+\operatorname{daim}(G) \leq 2 \delta(G)+2$.

\section{HOP HUB POLYNOMIAL OF GRAPHS}

DEFINITION 7. The hop hub polynomial of a connected graph $G$ of order $n$ is the polynomial

$$
H_{h}(G, x)=\sum_{i=h_{h}(G)}^{|V(G)|} h_{h}(G, i) x^{i},
$$

where $h_{h}(G, i)$ denotes the number of hop hub sets of $G$ of cardinality $i$ and $h_{h}(G)$ is the hop hub number of $G$.

To show this polynomial, we discuss this example.

EXAMPLE 2. Let $G$ be a graph as shown in Figure 2.

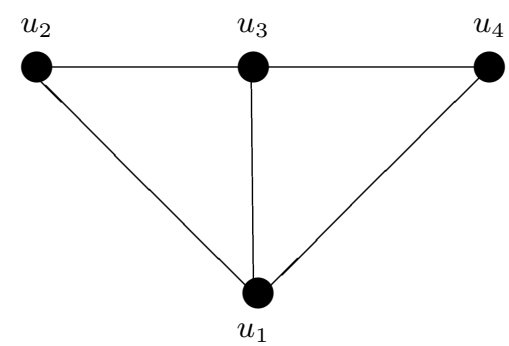

Fig. 2: Graph $(G)$

We have $h_{h}(G)=3$ such that $S_{1}=\left\{u_{1}, u_{2}, u_{3}\right\}, S_{2}=$ $\left\{u_{1}, u_{2}, u_{4}\right\}, S_{3}=\left\{u_{1}, u_{3}, u_{4}\right\}$ and $S_{4}=\left\{u_{1}, u_{2}, u_{3}, u_{4}\right\}$, are $H_{h}$-sets of $G$.

Then, $H_{h}(G, x)=3 x^{3}+x^{4}$.

TheOrem 8. Let $G$ be a path $P_{n}$. Then

$$
H_{h}\left(P_{n}, x\right)= \begin{cases}x^{2} & \text { if } n=2, \\
2 x^{2}+x^{3} & \text { if } n=3, \\
4 x^{2}+4 x^{3}+x^{4} & \text { if } n=4, \\
7 x^{3}+5 x^{4}+x^{5} & \text { if } n=5, \\
x^{n}+n x^{n-1}+\left(\left(\begin{array}{c}
n \\
2
\end{array}\right)-2\right) x^{n-2} & \text { if } n \geq 6 .\end{cases}
$$

Proof. We have the following cases:

Case 1: When $\mathrm{n}=2$. Let $S=\left\{v_{1}, v_{2}\right\}$ is $H_{h}$ set of $P_{2}$, hence $h_{h}\left(P_{2}\right)=2$. By definition of hop hub polynomial,

$$
H_{h}\left(P_{2}, x\right)=\sum_{i=2}^{2} h_{h}\left(P_{2}, i\right) x^{i}=x^{2} .
$$

Case 2: When $n=3$. Let Consider $V\left(P_{3}\right)=\left\{v_{1}, v_{2}, v_{3}\right\}$ and $h_{h}\left(P_{3}\right)=2$. Also every subset $S$ of vertex set of $P_{3}$ consisting of 2 elements, let it be $S_{1}=\left\{v_{1}, v_{2}\right\}$ and $S_{1}=\left\{v_{2}, v_{3}\right\}$, clearly, the number of sets that contain tow elements are two sets. Also the 
number of sets that contain three elements is only one set. Then by definition of hop hub polynomial, we have

$$
H_{h}\left(P_{3}, x\right)=\sum_{i=2}^{3} h_{h}\left(P_{3}, i\right) x^{i}=2 x^{2}+x^{3} .
$$

Case 3: When $n=4$, Consider $V\left(P_{4}\right)=\left\{v_{1}, v_{2}, v_{3}, v_{4}\right\}$ and $h_{h}\left(P_{4}\right)=2$. Every subset $S$ of vertex set of $P_{4}$ consisting of 2 elements, let it be $S_{1}=\left\{v_{1}, v_{2}\right\}, S_{2}=\left\{v_{3}, v_{4}\right\}$, $S_{3}=\left\{v_{1}, v_{4}\right\}$ and $S_{4}=\left\{v_{2}, v_{3}\right\}$, note that the number of sets that contain tow elements are four sets. We also note that the number of sets that contain three elements is only four set $\left(\begin{array}{l}4 \\ 3\end{array}\right)=4$. Note that the number of sets that contain four elements are one set. Then by definition of hop hub polynomial $H_{h}\left(P_{4}, x\right)=\sum_{i=2}^{4} h_{h}(G, i) x^{i}=4 x^{2}+4 x^{3}+x^{4}$.

Case 4: When $n=5$ Let Consider $V\left(P_{5}\right)=\left\{v_{1}, v_{2}, v_{3}, v_{4}, v_{5}\right\}$ and $h_{h}\left(P_{5}\right)=3$. Also every subset $S$ of vertex set of $P_{5}$ consisting of 3 elements is $\left(\begin{array}{l}5 \\ 3\end{array}\right)=10$ but the sets $S_{1}=\left\{v_{2}, v_{4}, v_{5}\right\}, S_{2}=$ $\left\{v_{1}, v_{2}, v_{4}\right\}$ and $S_{3}=\left\{v_{1}, v_{3}, v_{5}\right\}$ are not hop hub set, then every subset $S$ of vertex set of $P_{5}$ consisting of 3 elements is 7 . We note that the number of sets that contain four elements are five sets such that $\left(\begin{array}{l}5 \\ 4\end{array}\right)=5$. Note that the number of sets that contain five elements are one set. Then by definition of hop hub polynomial $H_{h}\left(P_{5}, x\right)=$ $\sum_{i=2}^{5} h_{h}(G, i) x^{i}=7 x^{3}+5 x^{4}+x^{5}$.

Case 5: When $n \geq 6$. Let $P_{n}=\left\{v_{1}, v_{2} \ldots, v_{n}\right\}$ be a path. Then we have $h_{h}\left(P_{n}\right)=n-2$. Also every subset of vertex set of $P_{n}$ consisting of $n-2$ elements and all its super sets form a hop hub set for the path $P_{n}$. Hence

$$
\begin{aligned}
& h_{h}\left(P_{n}, n-2\right)=\left(\begin{array}{c}
n \\
n-2
\end{array}\right)-2=\left(\begin{array}{c}
n \\
2
\end{array}\right)-2 . \\
& h_{h}\left(P_{n}, n-1\right)=\left(\begin{array}{c}
n \\
n-1
\end{array}\right)=\left(\begin{array}{l}
n \\
1
\end{array}\right)=n . \\
& h_{h}\left(P_{n}, n\right)=1 . \quad \square
\end{aligned}
$$

THEOREM 9. Let $G$ be a cycle $C_{n}$. Then

$$
H_{h}\left(C_{n}, x\right)= \begin{cases}x^{3} & \text { if } n=3, \\
4 x^{2}+4 x^{3}+x^{4} & \text { if } n=4, \\
18 x^{3}+15 x^{4}+6 x^{5}+x^{6} & \text { if } n=6, \\
-n x^{n-3}+\sum_{i=n-3}^{n}\left(\begin{array}{c}
n \\
n-3
\end{array}\right) x^{n-3} & \text { if } n=5, n \geq 7 .\end{cases}
$$

Proof. Case 1: When $n=3$. Suppose that $V\left(C_{3}\right)=$ $\left\{v_{1}, v_{2}, v_{3}\right\}$ and $h_{h}\left(C_{3}\right)=3$. It is clear that, the number of sets that contain three element is only one set. So by definition of hop hub polynomial, $H_{h}\left(C_{3}, x\right)=x^{3}$.

Case 2: When $n=4$. Consider $V\left(C_{4}\right)=\left\{v_{1}, v_{2}, v_{3}, v_{4}\right\}$ and $h_{h}\left(C_{4}\right)=2$. Also every hop hub subset $S$ of vertex set of $C_{4}$ consisting of 2 elements. let it be $S_{1}=\left\{v_{1}, v_{2}\right\}, S_{2}=\left\{v_{2}, v_{3}\right\}, S_{3}=$ $\left\{v_{3}, v_{4}\right\}$ and $S_{4}=\left\{v_{1}, v_{4}\right\}$. Also every hop hub sub set $S$ of vertex set of $C_{4}$ consisting of 3 elements. let it be $S_{1}=\left\{v_{1}, v_{2}, v_{3}\right\}$, $S_{2}=\left\{v_{2}, v_{3}, v_{4}\right\}, S_{3}=\left\{v_{3}, v_{4}, v_{1}\right\}$ and $S_{4}=\left\{v_{4}, v_{1}, v_{2}\right\}$, so the number of sets that contain three element are four sets. Also the number of sets that contain four element is only one set. Then by definition of hop hub polynomial $H_{h}\left(P_{4}, x\right)=4 x^{2}+4 x^{3}+x^{4}$. Case 3: When $n=6$. Consider $V\left(C_{6}\right)=\left\{v_{1}, v_{2}, v_{3}, v_{4}, v_{5}, v_{6}\right\}$ and $h_{h}\left(C_{6}\right)=3$. Also every subset of vertex set of $C_{6}$ consisting of 3 elements except subset $S_{1}$ and $S_{2}$ as in the following Figure 3 and all its super sets form a hop hub set for the cycle $C_{n}$. Hence $h_{h}\left(C_{6}, 3\right)=\left(\begin{array}{l}6 \\ 3\end{array}\right)-2=20-2=18$.

$h_{h}\left(C_{6}, 4\right)=\left(\begin{array}{l}6 \\ 4\end{array}\right)=15$.
$h_{h}\left(C_{6}, 5\right)=\left(\begin{array}{l}6 \\ 5\end{array}\right)=6$.

$h_{h}\left(C_{6}, 6\right)=\left(\begin{array}{l}6 \\ 6\end{array}\right)=1$.

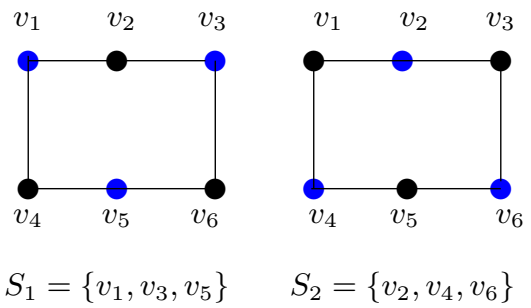

Fig. 3: Graph $\left(C_{6}\right)$

Case 4:When $n=5$ and $n \geq 7$. Let $C_{n}=\left\{v_{1}, v_{2}, \ldots, v_{n}\right\}$ be a cycle. Then we have $h_{h}\left(C_{n}\right)=n-3$. Also every subset of vertex set of $C_{n}$ consisting of $n-3$ elements and all its super sets form a hop hub set for the cycle $C_{n}$. Hence

$h_{h}\left(C_{n}, n-3\right)=\left(\begin{array}{c}n \\ n-3\end{array}\right)-n$.

$h_{h}\left(C_{n}, n-2\right)=\left(\begin{array}{c}n \\ n-2\end{array}\right)$.

$h_{h}\left(C_{n}, n-1\right)=\left(\begin{array}{c}n \\ n-1\end{array}\right)$.

$h_{h}\left(C_{n}, n\right)=\left(\begin{array}{l}n \\ n\end{array}\right)=1$.

THEOREM 10. For the complete graph $K_{n}, H_{h}\left(K_{n}, x\right)=x^{n}$.

Proof. Since $h_{h}\left(K_{n}\right)=n$, and $h_{h}\left(K_{n}, n\right)=1$. We have $H_{h}\left(K_{n}, x\right)=x^{n}$.

Proposition 4. Let $G \cong \overline{K_{n}}$. Then $H_{h}\left(K_{n}, x\right)=x^{n}$.

THEOREM 11. For the star graph $K_{1, n-1}$,

$$
H_{h}\left(K_{1, n-1}, x\right)=\sum_{i=1}^{n-1}\left[\left(\begin{array}{c}
n \\
i
\end{array}\right)-\left(\begin{array}{c}
n-1 \\
i
\end{array}\right)\right] x^{i}+x^{n}
$$

PROOF. Let $V\left(K_{1, n-1}\right)=\left\{v, v_{1}, v_{1}, \ldots, v_{n-1}\right\}$ is the vertices of $K_{1, n-1}$ and $v$ is the central vertex of $K_{1, n-1}$, since $H_{h}=$ $\left\{v, v_{i}\right\}, 1 \leq i \leq n-1$, then every hop hub set of cardinality $i$ must include the vertex $v$. The number of hop hub sets of cardinality 2 is $\left(\begin{array}{l}n \\ 2\end{array}\right)-\left(\begin{array}{c}n-1 \\ 2\end{array}\right)$. The number of hop hub sets of cardinality 3 is $\left(\begin{array}{c}n \\ 3\end{array}\right)-\left(\begin{array}{c}n-1 \\ 3\end{array}\right)$. So the number of hop hub sets of cardinality $i$ is $\left(\begin{array}{c}n \\ i\end{array}\right)-\left(\begin{array}{c}n-1 \\ i\end{array}\right), i \leq n-1$. Therefore,

$$
H_{h}\left(K_{1, n-1}, x\right)=\sum_{i=1}^{n-1}\left[\left(\begin{array}{c}
n \\
i
\end{array}\right)-\left(\begin{array}{c}
n-1 \\
i
\end{array}\right)\right] x^{i}+x^{n}
$$

\section{CONCLUSION}

In this paper, we have computed the concept of hop hubtic number of some standard graphs. Also, hop hub polynomial of some graph is obtained. $h_{\xi}(G)$ and $H_{h}(G, x)$ of several other families of graphs are an open problem.

\section{REFERENCES}

[1] S. Akbari, S. Alikhani and Y. H. Peng, Characterization of graphs using domination polynomial, European J., 31 (2010), 1714-1724. 
[2] G. D. Birkhoff and D. C. Lewis, Chromatic polynomials, Trans. Amer. Math. Soc., 60 (1946), 355-451.

[3] E. J. Cockayne, S. T. Hedetniemi, Towards a theory of domination in graphs, Networks 7 (1977), 247261.

[4] E. J. Farrell, A note on the clique polynomial and its relation to other graph polynomials, J. Math. Sci., (Calcutta) 8 (1997), 97-102.

[5] F. Harary, Graph theory, Narosa Publishing House, New Delhi, 2001.

[6] T. W. Haynes, S. T. Hedetniemi and P. J. Slater, Fundamentals of domination in graphs, Marcel Dckker, New York, 1998.

[7] S. I. Khalaf, V. Mathad and S. S. Mahde, Hubtic number in graphs, Opuscula Math., 38 (6) (2018), 841-847.

[8] S. S. Mahde and V. Mathad, Hub-integrity polynomial of graphs, TWMS J. App. Eng. Math., 10(2) (2020), 434-442.

[9] S. S. Mahde and A. S. Sand, Hop hub-integrity of graphs, Int. J. Math. And Appl., 9(4)(2021), 91-100.

[10] A. Mowshowitz, The characteristic polynomial of a graph, $J$. Combin. Theory Ser., B 12 (1972), 177-193.

[11] A. S. Sand, S. S. Mahde, Hop hub number in graphs, submitted.

[12] W. T. Tutte, A contribution to the theory of chromatic polynomials, Canad. J. Math., 6 (1954), 80-91.

[13] R. P. Veettil and T. V. Ramakrishnan, Introduction to hub polynomial of graphs, Malaya Journal of Matematik., 8(4) (2020) 1592-1596.

[14] M. Walsh, The hub number of graphs, Int. J. Math. Comput. Sci., 1 (2006), 117-124. 